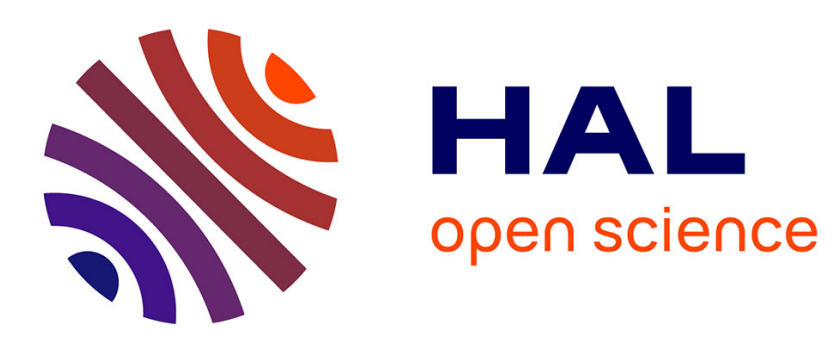

\title{
Filtering of matter symmetry properties by circularly polarized nonlinear optics
}

Julien Duboisset, Hervé Rigneault, Sophie Brasselet

\section{To cite this version:}

Julien Duboisset, Hervé Rigneault, Sophie Brasselet. Filtering of matter symmetry properties by circularly polarized nonlinear optics. Physical Review A: Atomic, molecular, and optical physics [1990-2015], 2014, 90, pp.063827. 10.1103/PhysRevA.90.063827 . hal-01231877

\section{HAL Id: hal-01231877 \\ https://hal.science/hal-01231877}

Submitted on 19 May 2016

HAL is a multi-disciplinary open access archive for the deposit and dissemination of scientific research documents, whether they are published or not. The documents may come from teaching and research institutions in France or abroad, or from public or private research centers.
L'archive ouverte pluridisciplinaire HAL, est destinée au dépôt et à la diffusion de documents scientifiques de niveau recherche, publiés ou non, émanant des établissements d'enseignement et de recherche français ou étrangers, des laboratoires publics ou privés. 


\title{
Filtering of matter symmetry properties by circularly polarized nonlinear optics
}

\author{
Julien Duboisset, ${ }^{*}$ Hervé Rigneault, and Sophie Brasselet \\ Aix-Marseille Université, CNRS, Centrale Marseille, Institut Fresnel, UMR 7249, 13013 Marseille, France
}

(Received 4 September 2014; published 17 December 2014)

\begin{abstract}
We propose a direct readout of symmetry information in matter using nonlinear optics. From combinations of circularly and longitudinally polarized optical fields, we construct irreducible spherical field tensors for secondand third-order nonlinear processes. The coupling of these field tensors to the matter susceptibility tensors allows filtering out of the susceptibility symmetries independently of the sample orientation in the laboratory frame. Experimental demonstrations are conducted on microcrystals, in a microscopy configuration compatible with symmetry order imaging.
\end{abstract}

DOI: 10.1103/PhysRevA.90.063827

PACS number(s): 42.65.An

\section{INTRODUCTION}

Acquiring structural information in an assembly of atoms and molecules requires the knowledge of its symmetry elements, which are fundamental building blocks of matter. Using symmetry as an observable appears therefore as an optimal way to probe the structural properties of complex materials and biological processes, which would be moreover interesting to obtain at high spatial and temporal resolution. Reading out of symmetry properties of matter is possible using nonlinear optics, taking advantage of the intrinsic relation between symmetry and nonlinear susceptibilities [1-3]. Second-order harmonic generation (SHG), for instance, is known to be allowed only in materials without symmetry inversion, whereas third-order nonlinearities, such as thirdorder harmonic generation (THG), four-wave mixing (FWM), or coherent Raman scattering (CRS) can occur in materials belonging to isotropic or centrosymmetric crystallographic groups [4]. Moreover, nonlinear optics offers the possibility of probing high-order symmetry information, thanks to the multiple fields' coupling mechanisms involving a large number of degrees of freedom in terms of light polarization states [5].

A large amount of work has been carried out to relate formally the probed nonlinear susceptibility tensors with the symmetry properties of matter $[1,6,7]$. Nonlinear Cartesian tensors can be expanded in their spherical representation in such a way that their components transform under proper rotations similarly to spherical harmonics of symmetry degree $l$ and order $m$ [2,8-10]. Following this spherical representation, harmonic light scattering signals in liquids have been used to extract the symmetry components of molecular nonlinear hyperpolarizability tensors $[1,3,11]$, allowing the development of an original molecular engineering strategy that has led to materials with optimized nonlinear responses $[12,13]$. While successful for understanding molecular-scale properties, this approach has apparently not yet been applied to molecular assemblies: second-order [14,15] and third-order [16-18] Cartesian susceptibility tensors of molecular materials and biological samples are usually probed by sets of linear polarization states, which is time consuming and necessitates disentangling complex Cartesian expressions to retrieve information on molecular orientations and symmetry orders.

\footnotetext{
*julien.duboisset@fresnel.fr
}

Accessing symmetry information in a direct way in ordered media is possible, provided that the spherical irreducible tensorial description is applied not only to matter but also to the electromagnetic fields. Within this representation, it is even possible to envision the filtering out of specific symmetry orders of matter, selecting proper sets of exciting fields. First proposed in Raman scattering [19] and later in the context of optical poling in organic materials [11], this approach has apparently not yet been exploited in the general frame of nonlinear optics. Here we apply the spherical irreducible tensorial description to both fields and matter in the cases of second-order and third-order nonlinear optical processes. Using fields with circular polarization states, we demonstrate the possibility of directly accessing in-plane sample symmetry, regardless of its orientation in the laboratory frame. We apply symmetry filtering to SHG and FWM microscopy imaging in micron-scale crystals of different symmetries, and show its potential to create additional contrast mechanisms.

\section{THEORY}

\section{A. Filtering process}

The electric nonlinear polarization is defined by the constitutive relationship

$$
\vec{P}=\epsilon_{0} \bar{\chi}^{(n)}: \vec{E}_{1} \otimes \cdots \otimes \vec{E}_{n},
$$

where $\bar{\chi}^{(n)}$ denotes the nonlinear susceptibility tensor of order $n, \vec{E}_{i, \ldots, n}$ are the vectorial fields involved in the nonlinear interaction, and $\otimes$ denotes the tensorial product. Exhibiting the norm of $\vec{P}$ and the complex unitary vectors $\hat{e}_{i}$ such as $\vec{P}=P_{0} \hat{e}_{0}$, and $\vec{E}_{i}=E_{i} \hat{e}_{i}$, Eq. (1) becomes

$$
P_{0}=\epsilon_{0}\left(E_{1}, \ldots, E_{n}\right) \bar{\chi}^{(n)} \bullet\left[\hat{e}_{0}^{*} \otimes \hat{e}_{1} \otimes \cdots \otimes \hat{e}_{n}\right],
$$

where - denotes the tensorial scalar product and $\hat{e}_{0}^{*}$ is the complex conjugate of the dipole polarization. Here all the polarization dependencies are collected in a unique "polarization tensor" $[10,11]$

$$
\bar{F}^{(n)}=\hat{e}_{0}^{*} \otimes \hat{e}_{1} \otimes \cdots \otimes \hat{e}_{n},
$$

showing that $P_{0}$ is finally the projection of the susceptibility tensor $\bar{\chi}^{(n)}$ on the polarization tensor $\bar{F}^{(n)}$.

In what follows, we show how this projection leads to a filtering out of the information on matter symmetry. Since symmetry must be decoupled from any orientation information, we use the irreducible spherical formalism where 
tensors are expanded on an orthonormal basis exhibiting rotational invariance. This representation allows identification of tensorial quantities based on pure symmetry considerations rather than a complex mixture of orientation and symmetry, such as is found in a Cartesian basis [1,8]. An irreducible spherical $n$-order tensor is labeled by its symmetry order $l \leqslant$ $n+1$ and possesses $m=2 l+1$ independent components, related to the spherical harmonics $Y_{m}^{l}[8,20] .{ }^{1}$ The coefficient $m$ can moreover be interpreted as the symmetry order of the tensor into the sample plane $(x, y), z$ being the direction of propagation of the excitation and detected fields. In such a representation, $\bar{\chi}^{(n)}$ is decomposed on spherical-basis-like components $\bar{C}_{m}^{l}$, as introduced in [2,21], with amplitudes $\chi_{m}^{l}$

$$
\bar{\chi}^{(n)}=\sum_{l m} \chi_{m}^{l} \bar{C}_{m}^{l} .
$$

\section{B. Irreducible field tensor}

Similarly to the $\bar{\chi}^{(n)}$ tensor, the field tensor $\bar{F}^{(n)}$ can be expanded under this irreducible spherical formalism. To calculate the spherical components of $\bar{F}^{(n)}$, each individual field polarization $\hat{e}_{i}$ of Eq. (3) must be represented in a spherical basis preserving the rotational invariance. The development below details the buildup of irreducible spherical representations for second- and third-order processes. For $l=1$, the three independent vectors $\hat{e}_{m}$ with $m=(-1,0,1)$ are expressed in the Cartesian frame as [1]

$$
\begin{aligned}
\hat{e}_{m=+1} & =1 / \sqrt{2}\left(+i \hat{e}_{x}+\hat{e}_{y}\right), \\
\hat{e}_{m=-1} & =1 / \sqrt{2}\left(-i \hat{e}_{x}+\hat{e}_{y}\right), \\
\hat{e}_{m=0} & =-i \hat{e}_{z} .
\end{aligned}
$$

These field vector components coincide with the in-plane left circularly polarized field $(m=+1)$, right circularly polarized field $(m=-1)$, and axial polarization field along $z(m=0)$, which are all invariant through rotations around the $\hat{z}$ axis. The irreducible representation of higher-order field tensors, built from this rank-1 expression in Eq. (3), have been described in the context of nonlinear optics by Maker [1]. The first step to expressing the rank-3 polarization tensor is to couple three irreducible tensors of rank one1 using the Clebsch-Gordan coefficients. A second step is required to take into account the different way of coupling the fields with respect to the symmetry of index permutation, using the $6 j$ Wigner coefficients. We limit this study to symmetric tensors, in which all the index permutations are allowed. Following this operation, the spherical field tensor basis components involved in symmetric nonlinear second-order processes (rank 3) are given by

$$
\begin{aligned}
\bar{F}_{m}^{(2), l}= & \sum_{\substack{m_{0} m_{1} \\
m_{2}}} \sum_{m_{i} l_{i}} U_{l_{i}}^{l}\left(l_{2} m_{2} l_{1} m_{1} \mid l_{i} m_{i}\right)\left(l_{i} m_{i} l_{0} m_{0} \mid l m\right) \\
& \times\left[\hat{e}_{m_{0}} \otimes \hat{e}_{m_{1}} \otimes \hat{e}_{m_{2}}\right] \\
= & \sum_{m_{0} m_{1} m_{2}} \mathfrak{M}_{m_{0}, m_{1}, m_{2}}^{l}\left[\hat{e}_{m_{0}} \otimes \hat{e}_{m_{1}} \otimes \hat{e}_{m_{2}}\right]
\end{aligned}
$$

\footnotetext{
${ }^{1}$ Using the convention of Eq. (5), $\bar{F}_{m}^{l}$ is related to $Y_{m}^{l}$ by a phase factor $i^{l}$.
}

with $l_{0}=l_{1}=l_{2}=1 .\left(l_{i} m_{i} l_{j} m_{j} \mid l_{k} m_{k}\right)$ is the Clebsch-Gordan coefficient with $m_{k}=m_{i}+m_{j}$ and $\left|l_{j}-l_{i}\right| \leqslant l_{k}<l_{j}+l_{i}$. $U_{l_{i}}^{l}$ is the symmetric recoupling matrix introduced in [1,22], whose values are given in the Appendix. $\mathfrak{M}_{m_{0}, m_{1}, m_{2}}^{l}$ is a global coefficient encompassing all sums over $\left(l_{i}, m_{i}\right)$ indices, nonvanishing only for $m=m_{0}+m_{1}+m_{2}$. The irreducible field tensor spherical basis components for third-order processes (rank 4) is built in the same way. A symmetric tensorial product with a fourth polarization vector $\hat{e}_{3}$ is combined with Eq. (6), leading to

$$
\begin{aligned}
\bar{F}_{m}^{(3), l}= & \sum_{\substack{m_{0}, m_{1} \\
m_{2}, m_{3}}} \sum_{\substack{l_{i}^{\prime}, m_{i}^{\prime} \\
l_{i}, m_{i}}}\left(l_{3} m_{3} l_{2} m_{2} \mid l_{i}^{\prime} m_{i}^{\prime}\right)\left(l_{i}^{\prime} m_{i}^{\prime} l_{1} m_{1} \mid l_{i} m_{i}\right) \\
& \times\left(l_{i} m_{i} l_{0} m_{0} \mid l m\right) V_{l_{i}^{\prime}}^{l} U_{l_{i}}^{l_{i}^{\prime}}\left[\hat{e}_{m_{0}} \otimes \hat{e}_{m_{1}} \otimes \hat{e}_{m_{2}} \otimes \hat{e}_{m_{3}}\right] \\
= & \sum_{\substack{m_{0}, m_{1} \\
m_{2}, m_{3}}} \mathfrak{M}_{m_{m_{2}, m_{3}}^{l}}^{l_{m_{0}, m_{1}}}\left[\hat{e}_{m_{0}} \otimes \hat{e}_{m_{1}} \otimes \hat{e}_{m_{2}} \otimes \hat{e}_{m_{3}}\right],
\end{aligned}
$$

where $V_{l^{\prime}}^{l}$ is the symmetric recoupling matrix [22] detailed in the Appendix, and $\mathfrak{M}_{m_{2}, m_{3}}^{l}$ is a coefficient which is nonvanishing only for $m=m_{0}+m_{1}+m_{2}+m_{3}$.

\section{Effective nonlinear susceptibility}

Equations (6) and (7) show finally that the irreducible spherical tensors $\bar{F}_{m}^{(n), l}$ are built from all basis polarization field combinations $\left(m_{0}, \ldots, m_{n}\right)$ which verify $m=m_{0}+\cdots+m_{n}$, with weight coefficients $\mathfrak{M}_{m_{0}, \ldots, m_{n}}^{l}$, reported in Table I. Conversely, the tensor field combination in Eq. (3) can be derived for incoming circular or $z$-polarized fields

$$
\hat{e}_{m_{0}} \otimes \cdots \otimes \hat{e}_{m_{n}}=\sum_{l} \mathfrak{M}_{m_{0}, \ldots, m_{n}}^{l} \bar{F}_{m}^{l} .
$$

Using the orthonormality property of the irreducible spherical susceptibility tensor $\bar{C}_{m}^{l}$ and the irreducible spherical basis tensor $\bar{F}_{m}^{l}$, this leads to

$$
P_{0}=\epsilon_{0} \xi_{m}\left(E_{1} \cdots E_{n}\right)
$$

with

$$
\xi_{m}=\sum_{l}(-1)^{l} \chi_{m}^{l} \mathfrak{M}_{m_{0}, \ldots, m_{n}}^{l}
$$

where $\xi_{m}$ can be interpreted as the rank- $n$ effective susceptibility of the probed matter, labeled by its in-plane symmetry order $m=m_{0}+\cdots+m_{n}$. It encompasses the full $\chi_{m}^{l}$ components by a summation over all three-dimensional (3D) symmetry orders $l$. Note that the use of a $z$ axial polarization would provide enough degrees of freedom to disentangle in-plane versus $3 \mathrm{D}$ information.

This spherical expansion shows finally that the use of circular polarizations for the exciting and detection fields has the unique ability to singularize individual symmetry orders $m$ and therefore to filter out in-plane symmetry information of matter in a single experimental scheme. In contrast, the use of linear polarizations would lead to a mixture of $m$ orders that can be extracted only by sequential excitation and detection schemes involving different polarization state directions $[11,14,15,17]$. The inspection of the $\mathfrak{M}_{m_{0}, \ldots, m_{n}}^{l}$ values show 
TABLE I. $\mathfrak{M}_{m_{0}, \ldots, m_{n}}^{l}$ values relating the field polarization tensorial products (rows) and the spherical irreducible tensors $\bar{F}_{m}^{l}$ (columns). No value means zero. A square root sign is to be understood over every coefficient, e.g., $\mathfrak{M}_{m_{0}=0, m_{1}=1, m_{2}=1}^{l=3}=\sqrt{1 / 3}$. Due to the symmetric construction of the irreducible spherical tensor, permutations are allowed and, for instance $\hat{e}_{1} \otimes \hat{e}_{0} \otimes \hat{e}_{0}=\hat{e}_{0} \otimes \hat{e}_{1} \otimes \hat{e}_{0}=\sqrt{4 / 15} \bar{F}_{1}^{3}-\sqrt{1 / 15} \bar{F}_{1}^{1}$.

\begin{tabular}{|c|c|c|c|c|c|c|c|c|c|c|c|c|c|c|c|c|c|c|}
\hline$\hat{e}_{m_{0}}$ & $\hat{e}_{m_{1}}$ & $\hat{e}_{m_{2}}$ & $\hat{e}_{m_{3}}$ & $\bar{F}_{4}^{4}$ & $\bar{F}_{3}^{4}$ & $\bar{F}_{2}^{4}$ & $\bar{F}_{1}^{4}$ & $\bar{F}_{0}^{4}$ & $\bar{F}_{3}^{3}$ & $\bar{F}_{2}^{3}$ & $\bar{F}_{1}^{3}$ & $\bar{F}_{0}^{3}$ & $\bar{F}_{2}^{2}$ & $\bar{F}_{1}^{2}$ & $\bar{F}_{0}^{2}$ & $\bar{F}_{1}^{1}$ & $\bar{F}_{0}^{1}$ & $\bar{F}_{0}^{0}$ \\
\hline$e_{1}$ & $e_{1}$ & $e_{1}$ & $e_{1}$ & 1 & & & & & & & & & & & & & & \\
\hline$e_{0}$ & $e_{1}$ & $e_{1}$ & $e_{1}$ & & $1 / 4$ & & & & & & & & & & & & & \\
\hline$e_{-1}$ & $e_{1}$ & $e_{1}$ & $e_{1}$ & & & $1 / 28$ & & & & & & & $3 / 14$ & & & & & \\
\hline$e_{0}$ & $e_{0}$ & $e_{1}$ & $e_{1}$ & & & $1 / 7$ & & & & & & & $-1 / 42$ & & & & & \\
\hline$e_{-1}$ & $e_{0}$ & $e_{1}$ & $e_{1}$ & & & & $1 / 28$ & & & & & & & $1 / 21$ & & & & \\
\hline$e_{1}$ & $e_{0}$ & $e_{0}$ & $e_{0}$ & & & & $1 / 7$ & & & & & & & $-3 / 28$ & & & & \\
\hline$e_{-1}$ & $e_{1}$ & $e_{1}$ & $e_{-1}$ & & & & & $1 / 70$ & & & & & & & $4 / 63$ & & & $4 / 45$ \\
\hline$e_{-1}$ & $e_{1}$ & $e_{0}$ & $e_{0}$ & & & & & $4 / 70$ & & & & & & & $1 / 252$ & & & $-1 / 45$ \\
\hline$e_{0}$ & $e_{0}$ & $e_{0}$ & $e_{0}$ & & & & & $8 / 35$ & & & & & & & $-4 / 7$ & & & $1 / 5$ \\
\hline$e_{1}$ & $e_{1}$ & $e_{1}$ & & & & & & & 1 & & & & & & & & & \\
\hline$e_{0}$ & $e_{1}$ & $e_{1}$ & & & & & & & & $1 / 3$ & & & & & & & & \\
\hline$e_{-1}$ & $e_{1}$ & $e_{1}$ & & & & & & & & & $1 / 15$ & & & & & $4 / 15$ & & \\
\hline$e_{1}$ & $e_{0}$ & $e_{0}$ & & & & & & & & & $4 / 15$ & & & & & $-1 / 15$ & & \\
\hline$e_{1}$ & $e_{-1}$ & $e_{0}$ & & & & & & & & & & $1 / 10$ & & & & & $1 / 15$ & \\
\hline$e_{0}$ & $e_{0}$ & $e_{0}$ & & & & & & & & & & $2 / 5$ & & & & & $-3 / 5$ & \\
\hline
\end{tabular}

that in the case of nonlinear rank-3 processes (SHG, etc.), two symmetry orders are accessible: $\xi_{1}$ (dipolar contribution) and $\xi_{3}$ (octupolar contribution) [3], which both exhibit noncentrosymmetry as expected. Rank-4 processes (THG, FWM, CRS, etc.) give access to isotropic $\xi_{0}$, second $\xi_{2}$, and fourth $\xi_{4}$ orders. Table I can be used to identify which circular field combination leads to these orders, paying attention to the presence of conjugated fields in the nonlinear processes, which imply a change of the $m_{i}$ sign (since $\hat{e}_{m_{i}}^{*}=\hat{e}_{-m_{i}}$ ). For instance, the filtering process which extracts $\xi_{0}$ in Eq. (9) will require the set $\hat{e}_{-1} \hat{e}_{1} \hat{e}_{-1} \hat{e}_{1}$ according to Table I. But due to the complex conjugates of the emitted field $m_{0}$ and the Stokes field $m_{3}$ in FWM, the experimental set of polarizations used to probe the isotropic order $\xi_{0}$ must be $\hat{e}_{1} \hat{e}_{1} \hat{e}_{1} \hat{e}_{1}$. Using the same experimental set, the third-order harmonic generation is sensitive to $\xi_{2}$ instead of $\xi_{0}[23-25]$.

\section{EXPERIMENTAL RESULTS}

\section{A. Experimental setup}

To experimentally illustrate nonlinear light-matter symmetry filtering, microscopy experiments were conducted on microcrystals using circular polarizations. Microcrystals of 1,3,5-tricyano-2,4,6-tris ( $p$-diethyl-aminostyryl)bezene (TTB) [26] and $\mathrm{NaCl}$ were imaged using second-order (SHG) and third-order (FWM) nonlinear processes. The TTB crystals were obtained by evaporation [26] and $\mathrm{NaCl}$ crystals were prepared by spraying saturated salt water on a cover glass. The crystal samples were covered with oil for index-matching purposes. The setup for polarized SHG and FWM microscopy is based on previously reported works [14,17]. Briefly, the light is focused on the sample using an objective lens $(\times 40$ numerical aperture 0.6). Images are obtained by moving the sample with a piezoscanning stage and the emitted signal is collected in the forward direction. The circular excitation polarization states are implemented using a unique achromatic quarter-wave plate just before the objective, the control of its left- or right-circular nature being performed beforehand using appropriate half-wave plates in the excitation paths involved. The emitted light is analyzed using an achromatic quarter-wave plate oriented at $45^{\circ}$ with respect to a Wollaston prism which separates the right and left circularly polarized light on two avalanche photodiodes (APDs). We denote by $I_{m}$ the intensity obtained by a set of excitation and detection circular polarizations such as $m=m_{0}+\cdots+m_{n}$.

\section{B. Results}

Figure 1 shows $I_{1}$ and $I_{3}$ images obtained with SHG on TTB microcrystals of needle shapes. These molecular crystals are known to be octupolar crystals oriented perpendicularly to their major axis [26]. Due to the crystal orientations, the planar octupolar symmetry is perpendicular to the propagation axis, which leads to unidirectional symmetry in the sample plane. From the intensities $I_{1}$ and $I_{3}$ the ratio $\xi_{3} / \xi_{1}=\sqrt{I_{3} / I_{1}}$ is calculated for each pixel of the image and summarized in a histogram; see Fig. 1(d). Due to the field-invariant rotation along the $z$ axis, the information extracted is also expected
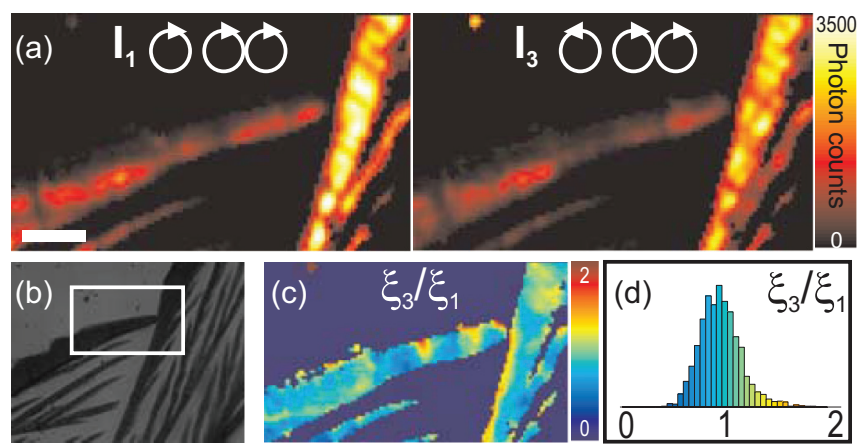

FIG. 1. (Color online) (a) SHG raw images of TTB crystals with dipolar $\left(\hat{e}_{1} \hat{e}_{1} \hat{e}_{1}\right)$ and octupolar $\left(\hat{e}_{-1} \hat{e}_{1} \hat{e}_{1}\right)$ polarization sets. (b) White light image and selected area for polarization-resolved SHG images. (c) Ratio between third and first orders (see text) and (d) its histogram. Scale bars: $10 \mu \mathrm{m}$. 


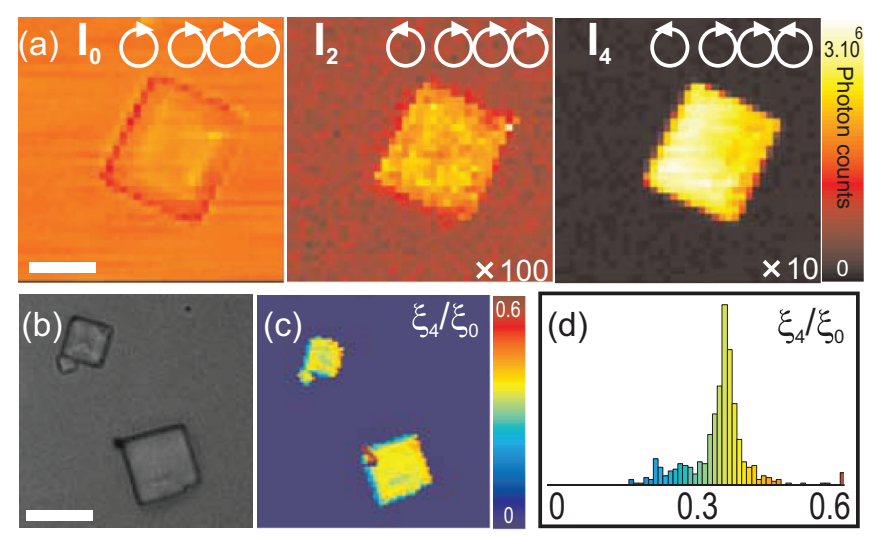

FIG. 2. (Color online) (a) FWM raw images of NaCl microcrystal surrounded by oil with its isotropic $\left(\hat{e}_{1} \hat{e}_{1} \hat{e}_{1} \hat{e}_{1}\right)$, second- $\left(\hat{e}_{-1} \hat{e}_{1} \hat{e}_{1} \hat{e}_{1}\right)$, and fourth-order $\left(\hat{e}_{-1} \hat{e}_{1} \hat{e}_{1} \hat{e}_{-1}\right)$ contributions. (b) White light image of three $\mathrm{NaCl}$ crystals with different orientations in the sample plane. (c) Ratio between fourth and zero orders and (d) its histogram. Scale bars: $10 \mu \mathrm{m}$.

to be invariant with respect to the orientation of the sample in the sample plane. Except for some specific regions which could be attributed to crystal defects, the value of $\xi_{3} / \xi_{1}$ is indeed confirmed to be homogeneous over the whole crystal. Moreover, the $\xi_{3} / \xi_{1}$ ratio is close to 1 , which leads to a similar weight for the dipolar and octupolar contributions, as expected for unidirectional crystals [11].

FWM images of $\mathrm{NaCl}$ salt microcrystals are depicted in Fig. 2. The different sets of circular polarizations lead to different information: the isotropic image $I_{0}$ shows a strong signal from the crystal and the oil. The $I_{2}$ image shows a very weak signal, two orders of magnitude smaller than $I_{0}$ (this residual signal is probably due to remaining imperfections in the circular excitation and detection states). Finally, the $I_{4}$ image shows a dominant, very strong signal in the $\mathrm{NaCl}$ crystals, while it is absent in the oil. This is expected from the fourfold symmetry of $\mathrm{NaCl}$, whereas the oil is an isotropic liquid. As previously, the $I_{4}$ magnitude does not depend on the crystal orientation, the ratio $\xi_{4} / \xi_{0}=\sqrt{I_{4} / I_{0}}$ being the same for all crystals present in the image; see Fig. 2(c). The $\xi_{4} / \xi_{0}$ ratio gives, moreover, quantitative information about the relative weight of fourth order and zero (isotropic) orders and is shown to be a fundamental symmetry property of $\mathrm{NaCl}$ crystals [17]. Note finally that the fourth-order measurement $\left(I_{4}\right)$ allows complete rejection of the $\mathrm{NaCl}$ isotropic contribution present in the crystals. This scheme, in addition to providing contrasted images, is a unique way to filter out symmetry orders without the need of postprocessing.

\section{CONCLUSION}

We have applied an irreducible spherical formalism to both fields and matter to express their coupling in nonlinear optics processes. Proper combinations of circular polarization fields were exhibited in order to filter out individually the accessible in-plane symmetry orders present in the probed matter. This readout process reveals quantitative symmetry information regardless of the in-plane orientation of the sample in the measurement plane. This scheme requires finally single, well-defined excitation and detection schemes, which makes it compatible with fast imaging of dynamical processes.

\section{ACKNOWLEDGMENTS}

The authors thank J. Zyss (ENS Cachan, France) for his important contribution to the start of this work. This work has been supported by Grant No. ANR-10-INBS-04-01 (France-BioImaging infrastructure network), Grant No. ANR11-INSB-0006 (France Life Imaging infrastructure network), and A*MIDEX project (Grant No. ANR-11-IDEX-0001-02), funded by the Investissements d'Avenir French Government program, managed by the French National Research Agency (ANR).

\section{APPENDIX: MATRICES $U$ AND $V$}

Here we give details of the matrices $U$ and $V$ for the complete symmetric set [1,22].

\begin{tabular}{cccccc}
\hline \hline$U_{l_{i}}^{l}$ & $l_{i}=0$ & $l_{i}=2$ & $V_{l_{i}}^{l}$ & $l_{i}=1$ & $l_{i}=3$ \\
\hline$l=1$ & $\sqrt{5 / 9}$ & $\sqrt{4 / 9}$ & $l=0$ & 1 & \\
$l=3$ & & 1 & $l=2$ & $\sqrt{7 / 10}$ & $\sqrt{3 / 10}$ \\
& & & $l=4$ & & 1 \\
\hline \hline
\end{tabular}

[1] P. D. Maker, Phys. Rev. A 1, 923 (1970).

[2] J. Jerphagnon, D. Chemla, and R. Bonneville, Adv. Phys. 27, 609 (1978).

[3] J. Zyss, J. Chem. Phys. 98, 6583 (1993).

[4] R. Boyd, Nonlinear Optics (Academic Press, San Diego, CA, 2008).

[5] S. Brasselet, Adv. Opt. Photonics 3, 205 (2011).

[6] J. L. Oudar, R. W. Smith, and Y. R. Shen, Appl. Phys. Lett. 34, 758 (1979).

[7] J. H. Christie and D. J. Lockwood, J. Chem. Phys. 54, 1141 (1971).

[8] J. A. R. Coope and R. F. Snider, J. Math. Phys. 11, 1003 (1970).

[9] Z. Ozgo and S. Kielich, Physica C 81, 151 (1976).
[10] R. Bonneville and D. S. Chemla, Phys. Rev. A 17, 2046 (1978).

[11] S. Brasselet and J. Zyss, J. Opt. Soc. Am. B 15, 257 (1998).

[12] Joseph Zyss and Isabelle Ledoux, Chem. Rev. 94, 77 (1994).

[13] C. Dhenaut, I. Ledoux, I. D. W. Samuel, J. Zyss, M. Bourgault, and H. Lebozec, Nature (London) 374, 339 (1995).

[14] J. Duboisset, D. Aït-Belkacem, M. Roche, H. Rigneault, and S. Brasselet, Phys. Rev. A 85, 043829 (2012).

[15] I. Gusachenko, Y. G. Houssen, V. Tran, J.-M. Allain, and M.-C. Schanne-Klein, Biophys. J. 102, 2220 (2012).

[16] F. Munhoz, H. Rigneault, and S. Brasselet, Phys. Rev. Lett. 105, 123903 (2010). 
[17] F.-Z. Bioud, P. Gasecka, P. Ferrand, H. Rigneault, J. Duboisset, and S. Brasselet, Phys. Rev. A 89, 013836 (2014).

[18] M. Zimmerley, P. Mahou, D. Débarre, M.-C. Schanne-Klein, and E. Beaurepaire, Phys. Rev. X 3, 011002 (2013).

[19] M. A. Yuratich and D. C. Hanna, Mol. Phys. 33, 671 (1977).

[20] G. Racah and U. Fano, Irreducible Tensorial Sets (Academic Press, New York, 1959).

[21] V. K. Khersonskiil, D. A. Varshalovich, and A. N. Moskalev, Quantum Theory of Angular Momentum (World Scientific, Singapore, 1988).
[22] H. A. Jahn and H. van Wieringen, Proc. R. Soc. London, Ser. A 209, 502 (1951).

[23] D. Oron, E. Tal, and Y. Silberberg, Opt. Lett. 28, 2315 (2003).

[24] N. Olivier, F. Aptel, K Plamann, M.-C. Schanne-Klein, and E. Beaurepaire, Opt. Express 18, 5028 (2010).

[25] Paul Kumar Upputuri, Jian Lin, Li Gong, Xiang-Yang Liu, Haifeng Wang, and Zhiwei Huang, Opt. Lett. 38, 1262 (2013).

[26] M. Y. Jeong, S. Brasselet, T. K. Lim, and B. R. Cho, Adv. Funct. Mater. 22, 788 (2012). 